Communication, technologies et développement

$5 \mid 2018$

Le numérique et le développement des Suds

\title{
l'Afrique entre communautés traditionnelles et monde virtuel
}

Africa between traditional communities and virtual world

África entre las communidades tradicionales y el mundo virtual

\section{Myriam Donsimoni}

\section{(2) OpenEdition}

\section{Journals}

Édition électronique

URL : http://journals.openedition.org/ctd/321

DOI : $10.4000 /$ ctd. 321

ISSN : 2491-1437

Éditeur

Chaire Unesco Pratiques émergentes en technologies et communication pour le développement

Référence électronique

Myriam Donsimoni, «l'Afrique entre communautés traditionnelles et monde virtuel », Communication, technologies et développement [En ligne], 5 | 2018, mis en ligne le 02 janvier 2018, consulté le 10 décembre 2020. URL : http://journals.openedition.org/ctd/321 ; DOI : https://doi.org/10.4000/ctd.321

Ce document a été généré automatiquement le 10 décembre 2020.

Communication, technologies et développement 


\title{
l'Afrique entre communautés traditionnelles et monde virtuel
}

\author{
Africa between traditional communities and virtual world \\ África entre las communidades tradicionales y el mundo virtual
}

Myriam Donsimoni

\section{NOTE DE L'AUTEUR}

\section{SWAHILI}

Muhtasari: Kuanzia kwa upinzani wa kitamaduni kwa cybercultures, miongo minne iliyopita ya karne ya ishirini wameona mabadiliko makubwa zaidi ya maisha ya kila siku. Ile mabadiliko inaweza kuelezewa kama mageuzi ya kawaida ya jamii za Magharibi, wale wanakawaida ya « kwenda haraka haraka »; sasa, jinsi gani tunaweza kuzingatia mageuzi ya nchi zinazoendelea, nchi iliyo na uchumi ulioharibiwa, na jamii iliyo kama jamii za jadi ? Katika mahadiko hili, tunashughulikia uwiano kati ya ile mawazo ya jamii, kama inavyohoneka pale pale Afrika, na mawazo wa digital ambayo huvutia na kuwahusisha zaidi na zaidi vijana. Je! Tunashuhudia maendeleo zaidi ya kiuchumi na kijamii kupitia matumizi ya chombo cha digital ? Waafrika wanajiunga zaidi jamii za jadi ambako wanabebaka tabia wao ya kuishi, utambulisho wao. Hatari ya jamii za jadi niku kosa ushirikiano ile watu wengine wanakemeaka asema inazuia maendelo. Internet na jamii ambazo virtueli (ya kule digital) wanabeba maana yao yote hapa, juu, wana fungua milango ya dunia na wana wesecha mawasiliano. Ile, jumuiya za jadi hazifanye

Maneno: Afrika, jadi, jamii, maendeleo

1 Des contre-cultures aux cybercultures, les quatre dernières décennies du $\mathrm{XX}^{\mathrm{e}}$ siècle ont été le théâtre des plus spectaculaires transformations de la vie quotidienne de l'histoire. Ces dernières peuvent être interprétées comme l'évolution normale des sociétés occidentales, qui sont plus ou moins habituées à aller vite ; mais comment les envisager dans les pays en voie de développement, marqués par une économie déstructurée et une société encore imbriquée dans les communautés traditionnelles? 
2 Dans cette étude, nous nous interrogeons sur la cohérence entre l'esprit communautaire, tel qu'il persiste en Afrique, et la culture numérique, qui attire et concerne de plus en plus de jeunes. Assistons-nous à une mise en dynamique vers plus de développement économique et social grâce à l'outil numérique, ou bien est-ce une ingérence occidentale supplémentaire dans un système non encore abouti qui ne pourra que s'en trouver encore plus déstructuré? Afin d'apporter des éléments de réponse à ces questionnements, nous examinerons dans un premier temps le rôle d'Internet dans le développement économique pour, dans un second temps, analyser les points de cohérence entre l'esprit communautaire, souvent hérité du passé, et la culture numérique, qui préfigure la société future.

\section{Internet et son rôle dans le développement économique}

3 Lorsqu'au début des années 1990, la croissance américaine battait son plein avec un taux de $4 \%$ entre 1994 et 1995, deux thèses se sont affrontées pour expliquer les causes de ce dynamisme: d'un côté celle du New Age, selon laquelle les NTIC étaient à l'origine du boom économique, de l'autre côté la thèse traditionnelle, qui avançait des arguments plus conjoncturels dans l'explication de la croissance et mettaient en avant le paradoxe de Solow: «L'âge de l'ordinateur est arrivé partout sauf dans les statistiques de la productivité ${ }^{1}$. Le cycle d'innovations qui a commencé avec l'arrivée d'Internet (nouvelle économie) ne serait pas porteur d'une croissance aussi importante que prévu². Certains expliquent ce paradoxe par les effets d'apprentissage et les coûts d'organisation. Tous les pays occidentaux, où le réseau internet est largement développé et utilisé, stagnent sur le plan économique depuis le choc de la crise qui a débuté en 2007 aux États-Unis.

4 Isoler l'impact de la nouvelle économie sur la croissance est un exercice difficile, dans la mesure où plusieurs transformations se sont produites avec l'arrivée des outils numériques. L'ordinateur et Internet ont à la fois des outils de production, de consommation et des moyens de communication; en ce sens, ils agissent à plusieurs niveaux. Une chose est sûre, l'ordinateur fait de plus en plus partie du quotidien du travailleur dans les pays industrialisés.

5 Le secteur numérique est en soi une filière porteuse en termes d'emploi et de compétence. La Toile contribue également au développement de secteurs connexes; elle influe sur la performance des entreprises, facilite la commercialisation et les relations entre parties prenantes, générant ainsi une dynamique de croissance. Une étude réalisée pour la France par McKinsey \& Company en $2010^{3}$ a montré qu'un euro investi dans les nouvelles technologies s'était traduit par deux euros de marge opérationnelle. Les PME en profitent plus particulièrement, car Internet leur facilite l'accès au marché et aux informations, supprimant un handicap par rapport aux grandes entreprises. L'étude en question a quantifié l'utilisation d'Internet à travers un «indice d'intensité web » et a montré que, de 2007 à 2010, les entreprises à forte «intensité web » ont crû deux fois plus vite que les autres ( $7 \%$ contre $3,2 \%$ ). Elles ont aussi exporté deux fois plus (réalisant en moyenne $4 \%$ de leur chiffre d'affaires à l'export) et ont créé le plus d'emplois.

6 L'une des raisons de ces résultats est l'apparition pour le client internaute d'un "surplus de valeur ", une valeur économique gratuite liée à l'utilisation des services dorénavant en ligne et financés par les annonces publicitaires. Le Web étant un marché presque parfait 
(atomicité des vendeurs et acheteurs, transparence de l'information, contexte pacifié, libre accès), la concurrence qui s'y joue conduit à un phénomène déflationniste dont le consommateur est le premier bénéficiaire. La vie de ce dernier s'est améliorée : facilité de la recherche, comparaison aisée des prix, accès à plus de savoirs, appartenance à une communauté virtuelle via les réseaux sociaux.

7 Sur le plan éducatif et culturel, Internet et le numérique constituent des outils formidables d'accès à l'information et à la connaissance, et il ne faut pas négliger son rôle sur le système éducatif, pilier du développement d'un pays.

Mais qu'en est-il dans les pays d'Afrique? L'Afrique subsaharienne affiche depuis 2008 une croissance de $5 \%$ par an en moyenne, sur laquelle la crise de 2009 n'a pas eu de prise ${ }^{4}$ . L'horizon s'est assombri avec la baisse des prix des matières premières (pétrole, métaux, minéraux, produits agricoles et alimentaires), qui représentent environ $82 \%$ des exportations de biens de la région. Après les records atteints en 2011, le repli des prix s'est accéléré au second semestre 2014 : - $35 \%$ pour les métaux de base et - $40 \%$ pour le pétrole entre janvier 2013 et mai $2015 ;-20 \%$ pour les produits alimentaires et $-5 \%$ pour les matières premières agricoles. L'étude sur l'Afrique subsaharienne réalisée en juin 2015 par la COFACE ${ }^{5}$ montre que les pays sont très inégaux face à la baisse des cours selon qu'ils sont exportateurs de matières premières non renouvelables (pétrole, métaux, minéraux) ou renouvelables (produits agricoles et alimentaires), et selon la plus ou moins grande diversification de leur économie. Certains tirent même remarquablement leur épingle du jeu. Plusieurs catégories de pays peuvent être distinguées :

1. les pays exportateurs nets de matières premières non renouvelables qui importent des produits alimentaires, et dont le solde commercial est fortement dégradé : Gabon, Nigeria, Congo, Angola ;

2. les pays exportateurs des deux catégories de biens (renouvelables et non renouvelables), modérément affectés par la baisse des prix en compensant les répercussions très négatives des baisses de prix du pétrole : Mozambique et Ghana ;

3. les pays exportateurs de produits agricoles et alimentaires et importateurs de pétrole, peu affectés, car la baisse du prix de leurs importations est supérieure à celle du prix de leurs exportations : Tanzanie ;

4. les pays engagés depuis plusieurs années sur le chemin de la diversification via le secteur manufacturier ou les services, qui s'en sortent particulièrement bien: Rwanda, Ouganda, Kenya et Éthiopie. Tous sont en Afrique de l'Est (si l'on exclut l'Afrique du Sud, diversifiée de longue date et très intégrée dans l'économie mondiale). Avec des taux de croissance supérieurs à $6 \%$, ces pays ont su valoriser leurs ressources agricoles grâce à une agroindustrie qui a permis à la fois d'augmenter la production locale et la valeur ajoutée, et de soutenir les emplois. Ils ont également su attirer des investisseurs étrangers (surtout chinois), en leur faisant miroiter des traitements de faveur. La montée en gamme de leur production industrielle est un atout nécessaire pour que l'activité progresse sur le long terme sans être pénalisée à court terme par le repli des matières premières.

Le déploiement du réseau internet en Afrique est en progression notable, mais le continent accuse toujours un important retard par rapport aux autres pays de la planète et une certaine lenteur d'accès au réseau. En 1994, deux pays seulement possédaient un réseau internet: l'Afrique du Sud et l'Égypte. L'une des explications à ce retard est sûrement liée à l'absence de langues africaines sur Internet ${ }^{6}$. La domination des langues européennes a limité la diffusion d'Internet en excluant ceux qui ne maîtrisaient pas complètement ces langues. La liste s'est toutefois rapidement allongée et, depuis 2000, la quasi-totalité des pays a un accès au Web. Le Maghreb dispose d'un réseau relativement 
bien connecté au reste du monde. En 2010, on y comptait 86217900 utilisateurs d'Internet, soit un taux de pénétration de plus de $8 \%$, ce qui correspond à $4,8 \%$ des utilisateurs dans le monde.

Les investisseurs du monde entier lorgnent l'Afrique et les projets d'équipement numérique vont bon train. Un projet récent et ambitieux envisage de connecter les pays africains au reste du monde grâce à un système de câble sous-marin (EASSy). Ce nouveau système permettra d'éviter que le trafic internet africain passe d'abord par l'Europe ou les États-Unis avant d'être réacheminé vers le continent, comme c'est le cas pour $75 \%$ du trafic africain actuel.

11 À partir des chiffres clés 2011 de l'Internet en Afrique, publiés par le Journal du Net ${ }^{7}$, nous avons repris les quatre groupes de pays précédemment listés pour repérer le nombre d'internautes (tabl. 1) et la part des internautes dans la population (tabl. 2) de ces pays, afin de voir si les pays les mieux équipés sont ceux dont la croissance est la plus élevée.

Tabl. 1 - Nombre d'internautes en 2011

\begin{tabular}{|c|c|c|c|}
\hline Pays & Nombre d'internautes & Rang Afrique/54 & Rang monde/195 \\
\hline \multicolumn{4}{|l|}{ GROUPE 1} \\
\hline Gabon & 122740 & 41 & 155 \\
\hline Nigéria & 46190431 & 1 & 10 \\
\hline Congo & 231826 & 30 & 138 \\
\hline Angola & 2898820 & 11 & 75 \\
\hline \multicolumn{4}{|l|}{ GROUPE 2} \\
\hline Ghana & 3522677 & 10 & 69 \\
\hline Mozambique & 1028977 & 16 & 106 \\
\hline \multicolumn{4}{|l|}{ GROUPE 3} \\
\hline Tanzanie & 5546218 & 6 & 50 \\
\hline \multicolumn{4}{|l|}{ GROUPE 4} \\
\hline Ouganda & 4490870 & 8 & 60 \\
\hline Kenya & 11650723 & 4 & 34 \\
\hline Ethiopie & 932077 & 18 & 110 \\
\hline Rwanda & 766006 & 20 & 114 \\
\hline Afrique du sud & 10596595 & 5 & 36 \\
\hline Pays du Maghreb & & & \\
\hline
\end{tabular}




\begin{tabular}{|l|l|l|l|}
\hline Algérie & 5037227 & 7 & 53 \\
\hline Maroc & 16459216 & 3 & 27 \\
\hline Tunisie & 4142276 & 9 & 61 \\
\hline Moyen-Orient & 29399597 & 2 & 18 \\
\hline Egypte & \multicolumn{3}{|l|}{} \\
\hline
\end{tabular}

Source : Myriam Donsimoni, à partir des chiffres du Journal du Net

Tableau 2 - Part des internautes dans la population en 2011

\begin{tabular}{|c|c|c|c|c|}
\hline Pays & $\begin{array}{l}\text { Part des internautes dans } \\
\text { la population }\end{array}$ & $\begin{array}{l}\text { Rang } \\
\text { Afrique/54 }\end{array}$ & $\begin{array}{l}\text { Rang } \\
\text { monde/195 }\end{array}$ & $\begin{array}{l}\text { Croissance } \\
2011-2012\end{array}$ \\
\hline \multicolumn{5}{|l|}{ GROUPE 1} \\
\hline Gabon & $8 \%$ & 23 & 141 & $5,7 \%$ \\
\hline Nigéria & $28,43 \%$ & 7 & 102 & $6,9 \%$ \\
\hline Congo & $5,60 \%$ & 28 & 147 & $7,2 \%$ \\
\hline Angola & $14,78 \%$ & 15 & 125 & $7,9 \%$ \\
\hline \multicolumn{5}{|l|}{ GROUPE 2} \\
\hline Ghana & $14,11 \%$ & 16 & 126 & $7,1 \%$ \\
\hline Mozambique & $4,30 \%$ & 32 & 155 & $7,4 \%$ \\
\hline \multicolumn{5}{|l|}{ GROUPE 3} \\
\hline Tanzanie & $12 \%$ & 20 & 131 & $7 \%$ \\
\hline \multicolumn{5}{|l|}{ GROUPE 4} \\
\hline Ouganda & $13,01 \%$ & 18 & 129 & $4,4 \%$ \\
\hline Kenya & $28 \%$ & 8 & 104 & $4,2 \%$ \\
\hline Ethiopie & $1,10 \%$ & 50 & 175 & $6,9 \%$ \\
\hline Rwanda & $7 \%$ & 26 & 144 & $7,7 \%$ \\
\hline $\begin{array}{l}\text { Afrique du } \\
\text { sud }\end{array}$ & $20,95 \%$ & 9 & 112 & $2,5 \%$ \\
\hline
\end{tabular}




\begin{tabular}{|c|c|c|c|c|}
\hline Algérie & $14 \%$ & 17 & 127 & $2,5 \%$ \\
\hline Maroc & $51 \%$ & 1 & 58 & $3,2 \%$ \\
\hline Tunisie & $38,81 \%$ & 3 & 80 & $3,3 \%$ \\
\hline \multicolumn{5}{|c|}{ Moyen-Orient } \\
\hline Egypte & $35,62 \%$ & 4 & 86 & $2,2 \%$ \\
\hline
\end{tabular}

Source : Myriam Donsimoni, à partir des chiffres du Journal du Net

12 L'échantillon de pays choisi ici fait ressortir une moyenne de $18,5 \%$ de la population ayant accès au réseau. L'Europe, en 2011, comptait environ 475 millions d'internautes, c'est-à-dire plus de $60 \%$ de sa population. L'Afrique est à la traîne, mais elle progresse dans son intérêt pour l'outil informatique et dans ses efforts pour élargir et améliorer l'accès au réseau internet.

13 Sur le total des pays d'Afrique que nous avons retenus sur la base des caractéristiques qui ressortent du rapport, 137470058 personnes utilisent Internet. Le Nigeria arrive en tête du nombre d'internautes en Afrique (suivi de l'Égypte) et au 7e rang pour la part des internautes dans la population (4e rang pour l'Égypte). Son taux de croissance entre 2011 et 2012 est relativement élevé, car il atteint 6,9\%, mais n'a pas fait reculer la pauvreté dans le pays ni réduit le taux de chômage, qui est de presque $24 \%^{8}$.

La prédominance des pays du Maghreb sur l'accès à Internet est visible à travers les chiffres des tableaux. Le Maroc est clairement celui qui présente la plus grande part de population connectée, avec $51 \%$ (plus que la Russie en 2011, avec $49 \%$ !). Il faut préciser que le modèle de développement adopté est fortement caractérisé par l'ouverture. Par contre, les difficultés économiques de l'Europe, son premier partenaire commercial, se sont traduites par une diminution de la croissance entre 2011 (4,6 \%) et 2012 (3,2 \%).

Le cas de l'Éthiopie mérite que nous nous y attardions, car c'est l'une des cinq économies les plus dynamiques du monde, avec une décennie d'expansion continue durant laquelle le PIB réel a augmenté de $10,8 \%$ en moyenne (selon le $\mathrm{FMI}^{9}$ ). Tous les secteurs de l'économie ont enregistré de bons résultats : l'agriculture (40,2\% du PIB) a progressé de $5,4 \%$, l'industrie ( $14 \%$ du PIB) de $21,2 \%$ et les services ( $46,2 \%$ du PIB) de $11,9 \%$. Des chiffres qui font rêver d'autant que le pays a su contenir l'inflation (7,1\% en 2014 contre $39,2 \%$ en 2011) et le déficit budgétaire (2,6\% du PIB en 2013-2014). Pourtant, seulement $1,10 \%$ de sa population a actuellement accès à Internet. La réussite économique éthiopienne s'appuie principalement sur des politiques publiques rigoureuses. Le gouvernement éthiopien a adopté un plan de croissance et de transformation visant à stimuler les secteurs agricole et industriel. La part de l'investissement public dans la FBCF est très importante : sur la période $2000-2012$, elle a dépassé les $65 \%^{10}$. Des restrictions à l'exportation ont été mises en place pour préserver les ressources naturelles et permettre au pays de développer des activités de transformation à plus forte valeur ajoutée (industrie du cuir). Cette réussite économique et ce dynamisme (croissance de 6,9\%) ont permis de mettre en réseau les établissements d'enseignement secondaire et supérieur. 
16 Le Ghana, le Mozambique, le Nigeria et la Tanzanie (à l'instar d'autres pays africains, et notamment d'Afrique de l'Ouest) ont également développé des services d'enseignement à distance et peuvent ainsi bénéficier des enseignements d'universités occidentales. On voit ici que l'impulsion vient des pouvoirs publics et que, si la mise en réseau accompagne le processus de croissance économique, elle n'en est pas à l'origine. Par contre, dans la plupart des pays, l'enseignement semble être le lieu de prédilection des implantations numériques et, en ce sens, Internet peut être perçu comme une impulsion à une dynamique d'ouverture et d'échanges d'étudiants, d'enseignants, d'idées et de thèmes de recherche, ce qui, à terme, structure les fondements d'une croissance endogène. Sur ce point, il est trop tôt pour faire le bilan; un recul est nécessaire, que l'actualité du phénomène ne permet pas encore. Cet investissement largement répandu en Afrique dans l'enseignement supérieur et le capital humain laisse toutefois présager une évolution dans le bon sens.

En Afrique du Sud, le Gouvernement a récemment lancé un programme pour faire d'Internet un véritable bien public. Les citoyens ont été consultés pour savoir comment ils souhaitaient que leur système de communications électroniques fonctionne. Cette ouverture au débat public rend accessible un domaine jusqu'ici réservé aux spécialistes de l'informatique, dans l'objectif ultime de réduire la pauvreté et de diffuser des informations sur la santé et le développement.

Sur le plan économique, une étude publiée en 2013 par le cabinet de conseil Dalberg Global Development Advisors ${ }^{11}$ sur l'Afrique subsaharienne présente l'impact potentiel d'Internet sur le développement dans l'agriculture, l'éducation, l'énergie, les finances, la gouvernance, la santé et les petites et moyennes entreprises (PME). Selon les conclusions de ce rapport, plus de $80 \%$ des chefs d'entreprise consultés espèrent qu'Internet les aidera à développer leurs affaires, réduira les coûts et créera des emplois. En matière de santé, le rapport indique que la formation à distance et l'e-learning ont contribué à remédier à la pénurie de travailleurs de la santé qualifiés en Afrique subsaharienne. Même dans le domaine de l'agriculture, l'accès à des informations en ligne sur les prix et la météo a permis d'améliorer la gestion de la chaîne d'approvisionnement, de réduire les coûts et d'améliorer le revenu des agriculteurs. Mais l'optimisation des répercussions d'Internet dépend de l'environnement réglementaire et des infrastructures haut débit dont peuvent disposer les pays : réglementations et infrastructures, deux conditions de réussite de l'outil internet. Le minimum pour profiter d'Internet est effectivement de disposer d'une alimentation électrique fiable. Dans des régions très pauvres et très éloignées sans accès à l'électricité, pas d'Internet! Mais là, les questions liées au développement passent par d'autres urgences et préoccupations que l'accès au réseau. C'est là qu'Internet creuse des inégalités déjà marquées.

Sur le plan économique, Internet accompagne la croissance, mais il ne fait pas le développement. Il peut être perçu comme un outil « occidental » de plus, au service d'un marché qu'il permet de rendre plus " parfait », car il offre un espace plus vaste et plus transparent de communication et d'échange d'informations (sur les prix entre autres), de confrontation de l'offre et de la demande. En fluidifiant les échanges, il constitue une institution incontournable dans la sphère économique et commerciale, et, en ce sens, il sert la croissance économique. Mais la croissance ne fait pas nécessairement le développement, et le marché ne fait pas la société ! 
21 Toutefois, Internet n'intervient pas uniquement dans la sphère économique ; son rôle dans la sphère des relations sociales est primordial. Est-ce la porte d'entrée par laquelle Internet peut plus largement contribuer au développement? Face à la communauté traditionnelle très forte en Afrique s'impose la communauté virtuelle. Se pose alors la question de la confrontation entre ces deux types de communautés.

\section{Esprit communautaire et culture numérique}

La question qui retient notre intérêt porte sur la relation entre les nouvelles technologies, relevant de la science et du modernisme, et le fonctionnement des groupes humains, marqués d'une idéologie et d'une doctrine sociale souvent encore enracinées dans les traditions et les coutumes. Internet est-il un moyen de passage de la communauté à la société, ou un autre type de communauté impliquant une coexistence? Dans l'un et l'autre cas, dans quelle mesure fait-il cohérence dans un contexte où l'essentiel fait encore défaut?

Ferdinand Tönnies ${ }^{12}$ distingue la volonté organique et la volonté réfléchie pour spécifier respectivement la communauté et la société. La première est liée au plaisir et à l'habitude. La seconde, dirigée vers l'échange et l'extériorisation de l'être, se manifeste par la réflexion et la décision. Elle marque la distinction avec le mode de fonctionnement de la communauté. La vie sociale gravite autour de l'échange, concrétisé par un contrat. La relation qui s'institue est de type externe et conventionnel, elle se vide de ses impulsions morales. Émile Durkheim ${ }^{13}$ aborde ce passage de la communauté à la société sous l'angle de la solidarité. Il distingue ainsi la solidarité mécanique et la solidarité organique. La solidarité organique se trouve dans la société moderne; elle permet de rapprocher des individus éloignés par la division du travail. La solidarité mécanique est caractéristique des situations de proximité propres aux sociétés traditionnelles et aux communautés. Il oppose dans sa démarche la différenciation à la similitude. La cohésion sociale s'appuie sur la transition de la mécanique à l'organisation. Plus tard, Georges Friedmann ${ }^{14}$ reprend cette distinction en différenciant le milieu naturel des campagnes et le milieu technique des villes. Dans le milieu naturel, l'homme réagit à des stimulations naturelles: la terre, l'eau, les plantes, les saisons, ou venues d'êtres vivants, animaux ou hommes. Le milieu technique se développe à partir de la révolution industrielle. Les stimulations naturelles cèdent leur place à un réseau de techniques de plus en plus sophistiquées.

Nous souhaitons partir de cette approche, qui s'attache à étudier le passage d'une structure simple à une structure plus complexe, pour étudier comment l'arrivée d'Internet modifie l'environnement habituel des sociétés africaines, encore pour beaucoup d'entre elles fortement marquées par le traditionalisme. Est-ce une manifestation de la transition dont parlent les sociologues selon un certain continuum ou, au contraire, est-ce un choc qui vient perturber ce passage ? Robert Redfield et Milton B. Singer ${ }^{15}$ remarquent que les sociétés ne peuvent être traitées comme le résultat d'un processus isolé ; elles subissent de multiples influences, des plus traditionnelles aux plus développées. Internet peut être considéré comme la manifestation d'une de ces influences, avec ceci de particulier qu'il est à l'interface entre les différentes sociétés, riches, pauvres, traditionnelles, modernes, pour peu que ces distinctions aient encore un sens. En ce sens, il traduit la coexistence de plusieurs modèles et, dans le nouvel environnement qu'il instaure, il peut être perçu soit comme l'« ami » qui rapproche, soit comme un « faux ami » qui fait oublier le sens des priorités. 
Nous proposons d'examiner le phénomène qui s'observe dans les pays du Nord, avec l'expansion actuelle d'une économie sociale et solidaire dont les communautés sont l'une des manifestations, et le phénomène qui caractérise plutôt les pays d'Afrique, où Internet est une intrusion supplémentaire de l'Occident, mais qui semble être plus recherchée et appropriée.

\section{Les communautés au Nord, des aires de repos}

Dans les sociétés des pays développés, le système capitaliste a substitué aux communautés l'attitude individualiste soi-disant efficace pour gagner la course à la croissance et au profit, permettre le bien-être. Ainsi, la disparition des communautés est associée à l'arrivée de la modernité et de l'individu moderne; elle correspond à une perte de socialisation et de convivialité. Au début, cette perte de convivialité a été compensée par la famille élargie et la mise en place d'organisations sociales sous forme d'associations professionnelles, de villages ou de quartiers, de sport et de loisirs, religieuses, caritatives, citoyennes, etc. Mais, progressivement, la famille s'est rétrécie, le capitalisme et la société de consommation ont laminé les organisations sociales et encouragé la consommation solitaire (voire même isolée, derrière son écran), au point que, dans les pays développés, on se trouve aujourd'hui en déficit de socialisation et de convivialité.

La chute du mur de Berlin en 1989 a relancé l'engouement contre la société telle qu'elle se présentait alors, avec un système technocratique et machiste marqué de multiples incohérences et incompétences. Des communautés diverses et variées virent le jour (hippie, punk, skinhead...) comme autant de symboles d'une volonté de contre-culture. Elles furent la manifestation de stratégies de révolte face à l'hégémonie d'un système sans valeur spirituelle profonde, où l'individualisme forcené conduisait paradoxalement à la perte des identités. Le processus de globalisation a amplifié ce ressenti et s'est accompagné d'une montée en pertinence d'un retour vers la proximité. Le niveau local et les spécificités territoriales ${ }^{16}$ interviennent alors de plus en plus dans la réalisation des performances productives et dans la construction des identités (l'origine territoriale d'un produit devient un argument de marketing). De nouvelles communautés (construites) émergent comme autant d'instruments d'une (re)socialisation à travers les diverses manifestations de l'économie sociale et solidaire. Elles ne consistent pas à revenir en arrière, mais à reconstruire des lieux où les besoins de socialisation et de convivialité peuvent être satisfaits. En ce sens, les communautés peuvent s'apparenter à de véritables refuges dans lesquels peut se ressourcer l'Homo sociabilis, qui a souffert de la montée en puissance de l'Homo ceconomicus. L'analogie avec les aires de repos des autoroutes nous semble parlante pour illustrer la contribution des communautés. Elles répondent à des besoins de pause nécessaires sur l'«autoroute" d'une modernité caractérisée non seulement par la rapidité, mais aussi par un sentiment d'enfermement et d'obligation d'aller dans le même sens que tout le monde.

Dans ce contexte, Internet simplifie le mécanisme des relations sociales (pas forcément leur contenu), mais n'annule pas la complexité du système économique global et contribue même à la rendre plus prégnante et d'autant plus impressionnante. La nouvelle économie basée sur la révolution numérique n'a pas permis de relancer la croissance, qui finit même par ne plus être une fin en soi (mouvement des "décroissants »). Il faudra composer de plus en plus avec les exigences sociales, et les outils numériques accompagnent cette tendance. 


\section{Les communautés au Sud, facteurs de résilience} communautés, et non d'individus; elles sont maillées d'innombrables solidarités qui, comme une toile d'araignée, leur donnent une très grande résilience. Elles contribuent à relier les morceaux d'une société déstructurée par la colonisation, par les exigences d'un capitalisme qui veut imposer des préconisations occidentales inadaptées, par des dirigeants le plus souvent incompétents et corrompus. Les communautés africaines traditionnelles sont les seules à permettre le fonctionnement correct de la sphère sociale, mais aussi de la sphère économique, car elles sont le support de l'entrepreneuriat. La décolonisation a produit des États faibles, car souvent artificiels et dotés de peu de légitimité ; ils ont été fragilisés par les modèles d'institutions politiques étrangères inadaptés. La pérennité des communautés traditionnelles est la manifestation d'un mouvement de survie. Elles sont le vestige d'un système qui permet aux identités, si différentes de celles que voulait imposer le consensus de Washington, de perdurer et de cimenter des relations économiques et sociales qui peuvent encore l'être et, par extension, de proposer un mode de fonctionnement adapté. Nous pourrions les trouver «traditionnelles », mais ne sont-elles pas finalement hors du temps?

communauté assure la cohésion sociale en permettant l'expression d'une volonté collective de résistance. Elle est la manifestation de la prise de conscience d'une identité et d'une spécificité. Certains auteurs ${ }^{17}$ notent toutefois que les communautés peuvent être à l'origine de tensions entre les groupes sociaux. Elles peuvent aussi constituer un obstacle au développement dans la mesure où, marquées par un traditionalisme fort, elles sont incompatibles avec l'innovation et l'esprit d'initiative ${ }^{18}$. En revanche, la communauté peut servir le développement en facilitant la participation et la mobilisation des populations au service des objectifs de ce dernier. En s'exprimant par des actions de proximité, elle prévient et résout des problèmes, favorise les partenariats et les coopérations, et permet de gérer la complexité parce qu'elle en connaît les ressorts. La communauté sert d'abord le développement d'un territoire, car elle suit une logique d'intervention "par le bas ", endogène et localisé ${ }^{19}$. Elle met en relation les sphères économique et sociale à travers le dialogue entre les entreprises et les territoires. En un mot, elle est le « plein » quand le marché est le « creux ».

31 L'intrusion d'Internet dans ce contexte est globalement bien perçue, car l'Afrique est caractérisée par une population très jeune ( $70 \%$ des moins de 30 ans dans le monde vivent en Afrique subsaharienne ${ }^{20}$ ), attirée par cet outil ludique et facile d'utilisation qui les ouvre sur le monde. On assiste aujourd'hui à une mutation sociale et culturelle qui se traduit par une profonde restructuration des modes de vie et des valeurs à la base du quotidien. Les jeunes incarnent l'émergence de nouveaux modèles (les "mutants » de Jean-Paul Gaillard ${ }^{21}$ ) et une nouvelle culture de la jeunesse se forge, qu'Internet a permis d'internationaliser et à laquelle chaque jeune de cette planète revendique de participer (depuis mai 1968), même si les contextes de par le monde ne sont pas les mêmes. Dans presque tous les pays, les jeunes ont préfiguré les différentes vagues du changement culturel à l'origine de l'idée de «village planétaire ${ }^{22} »$. Internet leur offre pour cela un outil formidable : il permet de diffuser une culture hédoniste à la place du puritanisme des pays du Nord et des traditionalismes et communautarismes du Sud. De nouveaux modèles de cohabitation voient le jour, remplaçant les modèles dépassés; la culture 
monolithique (par groupe ethnique ou classe sociale) est remplacée par une culture pluraliste et segmentée. D'une culture orale et écrite, on est passé à une culture visuelle et multimédia, du face-à-face à des relations virtuelles.

En 2011, le Printemps arabe a marqué un nouveau tournant dans les manifestations de contre-culture qui marquent les revendications d'une jeunesse qui ne trouve pas dans ce qui lui est proposé sujet à épanouissement. Mais la jeunesse africaine est à la charnière entre communautés traditionnelles et communautés virtuelles, contraintes et libertés, connaissance et inconnu, confiance et risque. Elle subit ce que l'anthropologue anglais Gregory Bateson ${ }^{23}$ a appelé le double bind (double contrainte) : d'une part, une crise culturelle qui remet en question les fondements idéologiques, religieux et moraux de la civilisation contemporaine, et d'autre part, l'émergence de nouveaux modèles qui annoncent le passage d'une société reposant sur l'économie industrielle et la culture moderne (éthique protestante et idées des Lumières) à une société basée sur l'économie de l'information et un post-modernisme aux contours encore diffus.

Margaret Mead ${ }^{24}$ aborde cette métamorphose sous l'angle des processus de transmission générationnelle. Elle distingue les cultures postfiguratives, cofiguratives et préfiguratives. Les cultures postfiguratives correspondent aux sociétés primitives et à des groupuscules religieux ou idéologiques, au sein desquels les enfants apprennent principalement de leurs aînés; le temps est répétitif et le changement social lent. Les cultures cofiguratives correspondent aux grandes civilisations basées sur l'État, dans lesquelles les enfants comme les adultes apprennent de leurs contemporains; le temps est moins cyclique et le changement social accéléré. Viennent enfin les cultures préfiguratives, qui ont émergé dans les années 1970 et où les jeunes assument une nouvelle autorité grâce à leur compréhension préfigurative d'un avenir encore inconnu. Cette dernière configuration nous semble primordiale, car, si elle a concerné pendant 20 ans certains milieux de la société occidentale, elle a subi un élan colossal grâce au numérique et à Internet. Les réseaux sociaux en ligne ont déclenché une dynamique où les jeunes du monde entier peuvent se reconnaître et s'affirmer. La toile leur permet de créer ou de rétablir de la proximité entre eux par le biais des communautés virtuelles. L' Homo sociabilis a trouvé un allié en l'Homo numericus! Tous les pays du monde, sur ce point-là, se trouvent au même niveau.

Une nouvelle humanité est en cours d'instauration, tiraillée encore par des doutes et des hésitations qui se traduisent par le malaise social généralisé que l'on connaît actuellement dans presque tous les pays du monde. José Ortega y Gasset analysait cela comme la coexistence de plusieurs présents ${ }^{25}$. L'enjeu réside dans les choix que les jeunes feront concernant leur mode de vie, ce qu'Amartya Sen appelle la « capabilité26 ».

\section{Communautés traditionnelles vs communautés virtuelles}

35 Les questions qui guident notre réflexion sur ce point sont les suivantes: peut-on assimiler communauté traditionnelle et communauté virtuelle dans leur contribution et que peut-on en attendre sur les plans économique et social?

Les communautés héritées traditionnelles présentent toujours un centre de référence fondateur de l'identité, une structure hiérarchique, une stratification sociale avec répartition des rôles et distribution des places. Elles sont régies par des règles (souvent 
tacites) très puissantes, qui ne sont pas remises en cause au nom d'un certain respect des traditions. Par contre, les communautés construites modernes, telles que les réseaux sociaux sur Internet, n'ont pas ces caractéristiques. Le propre d'une communauté est de définir un espace socio-économique décrivant un intérieur et un extérieur; en ce sens, elle est un ensemble d'individus, avec des frontières formalisées et visibles. Un réseau est plutôt un ensemble d'interactions n'ayant pas de frontière bien définie. La communauté est faite pour durer et pour être transparente, visible par ses membres comme par l'extérieur ; le réseau est plus occulte. La communauté est une totalité, avec des valeurs partagées et un fort sentiment d'appartenance. Le réseau est plutôt un processus émergent et dynamique, sa faible visibilité empêche la constitution d'appartenances fortes. La communauté induit pour ses membres un engagement vis-à-vis de sa totalité, une volonté d'agir tous ensemble. Le réseau, quant à lui, conduit à un engagement dans une ou plusieurs interactions. La transgression des règles de la communauté donne lieu à différentes sanctions selon le contexte et la situation, pouvant aller jusqu'à l'exclusion. Le réseau est plutôt un ensemble déstructuré et protéiforme.

Du point de vue de la théorie des systèmes qui analyse les propriétés de chaque type d'organisation, la propriété visée par la communauté est sa forte stabilité, sa résilience, c'est-à-dire sa capacité à perdurer en résistant aux pressions et forces extérieures. Le réseau, lui, est d'abord souple et flexible, sa vertu est sa capacité d'adaptation à un environnement changeant, sa capacité dynamique d'infiltration de nouveaux milieux. Selon la distinction faite par Mark Granovetter ${ }^{27}$, la communauté sera caractérisée par l'existence de liens forts entre ses membres, reposant sur une forte confiance et une importante complicité, construites sur un vécu et des valeurs communes. Le réseau est construit sur des liens faibles, qui engagent moins et intègrent moins de ressources, mais sont plus vite établis et donc plus nombreux et plus flexibles. D'une certaine manière, les deux structures sont conçues pour résister à l'environnement, mais la communauté protège d'abord son mode d'organisation, son fonctionnement, son mode de vie et l'esprit qui l'anime, alors que le réseau privilégie ses membres, quitte à modifier son organisation. De ce point de vue, la communauté est d'abord une structure sociale considérée comme un tout: elle représente en quelque sorte un bien commun pour chacun de ses membres. Le réseau reste une somme d'individus.

Deux risques principaux peuvent menacer les communautés. Le premier est celui d'une déviation holiste, qui se manifeste par un certain conservatisme économique. Il s'agit des situations où des initiatives individuelles et innovantes, entrepreneuriales ou institutionnelles, peuvent entrer en conflit avec la fonction de protection de la communauté ou avec sa fonction d'inclusion sociale. Le second risque est celui d'une déviation narcissique, où la communauté échange moins et entretient moins de relations avec l'extérieur, affichant une tendance au repli sur elle-même (communautarisme). Les réseaux ont également leurs faiblesses. Ils ont une forte connectivité et un pouvoir important de croissance et d'infiltration de nouveaux milieux, mais ils sont au contraire assez peu résilients et, en fait, très vulnérables. Le réseau est très dépendant de chacun de ses membres, et la défection de quelques individus peut le mettre entièrement à bas.

La communauté répond à un besoin de proximité. On parle d'abord de la proximité au sens géographique, c'est-à-dire de la possibilité de rencontrer physiquement les membres de la communauté. La proximité est importante comme composante de la convivialité : c'est le fait non seulement de connaître son entourage, mais aussi d'avoir près de soi une partie de ses proches. Elle répond à un besoin de transparence et de confiance qui permet 
d'aborder sereinement la vie quotidienne. Étant constituée de liens forts entre ses membres, la communauté devient naturellement un creuset d'identité collective. La communauté virtuelle d'Internet supplante la proximité physique, et la perception des relations et des personnes peut s'en trouver modifiée. Cette distanciation objective les relations et libère des contraintes du communautarisme traditionnel. Internet permet la liberté d'expression, mais la liberté sans l'égalité renvoie à la loi du plus fort (et c'est une situation injuste), et l'égalité sans la liberté correspond à un monde contre nature où tout le monde est au même niveau (en témoigne l'échec du système soviétique). Si l'on n'ajoute pas le terme de fraternité, on supprime le fondement de la citoyenneté. Se pose alors la question de savoir si ce fameux slogan issu de la Révolution française n'est pas une fois revendiqué au niveau international - un oxymore qu'Internet a mis en évidence et qui, faute de solution universelle, ne peut qu'apporter une désarticulation supplémentaire dans des sociétés déjà déstructurées.

Le lien que permet Internet avec le reste du monde est superficiel et volatile et s'oppose aux notions de confiance et de durabilité de la communauté. La première ne peut pas remplacer la seconde sous peine de déstabiliser les fondements d'un processus de développement, mais leur complémentarité permet de combler les insuffisances et inefficacités de systèmes qui imposent un individualisme dont la nature humaine ne peut se satisfaire.

\section{Conclusion}

41 Nous avons constaté dans un premier temps qu'Internet, s'il accompagne la croissance économique, ne peut en être à l'origine. Mais il est important pour resserrer les liens entre les personnes à travers les différentes communautés virtuelles qui se mettent en place dans le monde. Autant le système économique capitaliste est à l'origine d'inégalités et de problèmes sociaux, autant Internet met tout le monde à égalité derrière le clavier de l'ordinateur. L'inégalité persiste dans ce qu'il est convenu d'appeler la fracture numérique, c'est-à-dire les disparités d'accès aux outils informatiques et à Internet, mais cette fracture se comble rapidement grâce aux investisseurs qui mesurent l'enjeu que constitue l'équipement des zones encore défavorisées. Le secteur internet attire des investissements là où les investisseurs n'allaient plus, l'Afrique.

Les populations africaines sont encore fortement attachées aux communautés traditionnelles où elles puisent leur mode de vie, leur identité. Le risque inhérent aux communautés traditionnelles est un certain communautarisme que certains accusent d'entraver le processus de développement. Internet et les communautés virtuelles prennent ici tout leur sens, car, contrairement aux communautés traditionnelles, elles ouvrent les portes du monde et facilitent la communication. Mais il convient d'être vigilant face à deux dérives des réseaux virtuels : la difficulté de gérer une liberté à laquelle les personnes ne sont pas habituées et la superficialité des liens sur la Toile, qui masque les priorités. Ces deux risques peuvent constituer un facteur supplémentaire de déstructuration dans des économies déjà fragiles. Heureusement, entre les mains d'une jeunesse africaine de mieux en mieux éduquée, dans le cadre rassurant et stabilisant des communautés héritées, l'outil Internet bien utilisé peut servir les priorités d'un processus de développement décidé par les Africains eux-mêmes. 


\section{BIBLIOGRAPHIE}

Bateson Gregory, Steps to an Ecology of Mind, University of Chicago Press, Chicago, 2000 ( $1^{\text {re }}$ éd. 1972)»

Boumphrey Sarah, « Special Report : The World's Youngest Populations », Euromonitor International, 2012, disponible sur blog.euromonitor.com.

Chiffres clés de l'internet en Afrique : http://www.journaldunet.com/web-tech/chiffresinternet/afrique/continent-993

CNUCED, Catalyser l'investissement pour une croissance transformatrice en Afrique, Rapport sur le développement économique en Afrique, 2014, 113 pages.

Cohen Daniel, Le monde est clos et le désir est infini, Albin Michel, Paris, 2015.

Dalberg, «Impact of the Internet in Africa : Establishing Conditions for Success and Catalysing Inclusive Growth in Ghana, Kenya, Nigeria and Senegal », rapport de recherche, Dalberg, Dakar, 2013, disponible sur http://www.dalberg.com.

Durkheim Émile, De la division du travail social, Presses universitaires de France, coll. Quadrige,

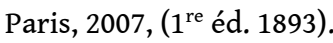

Fischer Georges, « Tradition, identité nationale et développement. Note introductive », Politique étrangère, vol. 40, n 6, 1975, p. 657-661, disponible sur http://www.persee.fr.

FMI, Perspectives économiques mondiales, rapport 2011.

Friedmann Georges (dir.), Villes et Campagnes. Civilisation urbaine et civilisation rurale en France, actes de la IIe Semaine sociologique (Paris, mars 1951), Armand Colin, coll. « Bibliothèque générale de l'École des hautes études en sciences sociales ", Paris, 1953.

Gaillard Jean-Paul, Enfants et adolescents en mutation. Mode d'emploi pour les parents, éducateurs, enseignants et thérapeutes, ESF Éditeur, coll. «L'Art de la psychothérapie », Issy-les-Moulineaux, 2009.

Granovetter Mark, « La force des liens faibles », dans Granovetter Mark, Sociologie économique, traduit de l'américain par This Saint-Jean Isabelle, Le Seuil, coll. «Économie humaine », Paris,

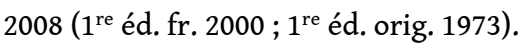

Laulan Anne-Marie, Lenoble-Bart Annie (dir.), Les Oubliés de l'Internet. Cultures et langues sur l'Internet, oubli ou déni ?, Les Études hospitalières, coll. « Science, éthique et société », Bordeaux, 2014.

McLuhan Marshall, The Medium is the Message : An Inventory of Effects, Bantam Books, New York / Londres / Toronto, 1967.

Mead Margaret, Culture and Commitment : The New Relationships Between the Generations in the 1970s, Columbia University Press, New York, 1978 (1re éd. 1970).

Ortega y Gasset José, Euvres complètes. Tome I : Qu'est-ce que la philosophie ? Leçons de métaphysique, traduit de l'espagnol par Lorvellec Yves et Pierre Christian, Klincksieck, Paris, 2000

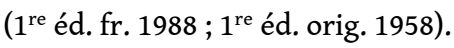


Pecqueur Bernard, «La logique de la proximité », Sciences humaines, hors-série $n^{\circ} 8$ (Régions et Mondialisation), 1995, p. 43-44.

Pecqueur Bernard, Le Développement local. Pour une économie des territoires, Syros, coll. « Alternatives économiques », Paris, 2000 ( $1^{\text {re }}$ éd. 1989).

Redfield Robert, Singer Milton B., « The Cultural Role of Cities », Economic Development and Cultural Change, vol. 3, no1 (The Role of Cities in Economic Development and Cultural Change), 1954, p. 53-73, disponible sur http://www.jstor.org.

Sen Amartya, Commodities and Capabilities, North-Holland, coll. « Professor Dr. P. Hennipman Lectures in Economics » (no7), Amsterdam / New York / Oxford, 1985.

Tönnies Ferdinand, Gemeinschaft und Gesellschaft. Grundbegriffe der reinen Soziologie, Wissenschaftliche Buchgesellschaft, Darmstadt, 2005 (1 $1^{\text {re }}$ éd. 1887).

Zimi Gutu Kia, Le Modèle Monade de développement. Le développement des communautés en Afrique, Author House, Bloomington, 2012.

\section{NOTES}

1. Déclaration de Robert Solow au New York Times Book Review, 1987.

2. Cohen, 2015.

3. McKinsey Global Survey Results : Innovation and commercialization, 2010.

4. Le Monde, juin 2015.

5. Panorama: Evolution du risque pays dans le monde, «Afrique subsaharienne : soleil à l'est, temps nuageux au centre », par les économistes du groupe COFACE.

6. Laulan, Lenoble-Bart (dir.), 2014.

7. http://www.journaldunet.com/web-tech/chiffres-internet/afrique/continent-993.

8. Cette analyse peut faire l'objet de certaines réserves quant à la fiabilité des chiffres et à leur interprétation mais elle s'appuie sur l'étude du Journal du Net, et nous n'avons aucun moyen de les remettre en cause.

9. Perspectives économiques mondiales, Rapport du FMI, 2011.

10. Rapport 2014 de la CNUCED sur le développement économique en Afrique.

11. Dalberg, 2013.

12. Tönnies, 2005 (1re éd. 1887).

13. Durkheim, (1re éd. 1893).

14. Friedmann (dir.), 1953.

15. Redfield, Singer, 1954.

16. Pecqueur, 1995.

17. Gutu Kia Zimi, 2012.

18. Fischer, 1975.

19. Pecqueur, 2000 (1re éd. 1989).

20. Boumphrey, 2012.

21. Gaillard, 2009.

22. McLuhan, 1967.

23. Bateson, 1972.

24. Mead, 1978 (1re éd. 1970).

25. Ortega y Gasset, 2000 (1re éd. fr. 1988 ; 1re éd. orig. 1958).

26. Sen, 1985.

27. Granovetter, 2008 ( $1^{\text {re }}$ éd. fr. $2000 ; 1^{\text {re }}$ éd. orig. 1973). 


\section{RÉSUMÉS}

Des contre-cultures aux cybercultures, les quatre dernières décennies du $\mathrm{XX}^{\mathrm{e}}$ siècle ont été le théâtre des plus spectaculaires transformations de la vie quotidienne. Ces dernières peuvent être interprétées comme l'évolution normale des sociétés occidentales, qui sont plus ou moins habituées à aller vite; mais comment les envisager dans les pays en voie de développement, marqués par une économie déstructurée et une société encore imbriquée dans les communautés traditionnelles?

Dans cette étude, nous nous interrogeons sur la cohérence entre l'esprit communautaire, tel qu'il persiste en Afrique, et la culture numérique, qui attire et concerne de plus en plus de jeunes. Assistons-nous à une mise en dynamique vers plus de développement économique et social grâce à l'outil numérique, ou bien est-ce une ingérence occidentale supplémentaire dans un système non encore abouti qui ne pourra que s'en trouver encore plus déstructuré?

Les populations africaines sont encore fortement attachées aux communautés traditionnelles où elles puisent leur mode de vie, leur identité. Le risque inhérent aux communautés traditionnelles est un certain communautarisme que certains accusent d'entraver le processus de développement. Internet et les communautés virtuelles prennent ici tout leur sens, car, contrairement aux communautés traditionnelles, elles ouvrent les portes du monde et facilitent la communication.

The last four decades of the XXth century have been the theater of the most spectacular transformations of the everyday life. These transformations can be interpreted as the normal evolution of the Western societies, which are used more or less to go fast; but how can we consider them in developing countries, that are marked by destructured economy and a society which is still embedded in traditional communities?

In this study, we wonder about the coherence between the community spirit, such as it persists in Africa, and the digital culture, which attracts and concerns more and more young people. Are we witnessing a new dynamic towards more economic and social development based on the digital tool, or is it an additional western intervention in the not yet accomplished system which can only be even more distructured.

African populations are still strongly attached to the traditional communities from where they draw their lifestyle, their identity. The risk inherenttothetraditionalcommunities is a certain communitarianism that is sometimes accused of hindering the process of development. Internet and the virtual communities take here all their sense, because, unlike the traditional communities, they open the doors to the world and facilitate communication.

\section{INDEX}

Mots-clés : Afrique, tradition, communauté, développement

Keywords : Africa, tradition, community, development 
AUTEUR

MYRIAM DONSIMONI

Université de Savoie Mont-Blanc 